\title{
Extended Application of Barley EST Markers for the Analysis of Alien Chromosomes Added to Wheat Genetic Background
}

\author{
Adel Abdel-Aziz Hagras'), Masahiro Kishii'1,3), Kazuhiro Sato'), Hiroyuki Tanaka ${ }^{1)}$ and Hisashi Tsujimoto*1) \\ 1) Laboratory of Plant Genetics and Breeding Science, Faculty of Agriculture, Tottori University, 4-101 Minami, Koyama, Tottori 680- \\ 8553, Japan \\ 2) Research Institute for Bioresources, Okayama University, 2-20-1 Chuo, Kurashiki, Okayama 710-0046, Japan \\ 3) Present address: Genetic Resources, CIMMYT, Apdo. Postal 6-641, 06600 Mexico, D.F., Mexico
}

The main objective of this study is to produce DNA markers of polymorphism between wheat and some important alien species. The applicability of 1,165 barley EST primer sets to amplify markers showing polymorphism between wheat and ten alien species, covering a wide range of variation in Triticeae, was investigated. These primers consisted of four series. Series 1 was randomly chosen from a pool of barley EST primer sets. The remaining three were pre-screened in a previous study and showed polymorphic and coamplified patterns between barley and wheat. From $22 \%$ to $100 \%$ of the primer sets amplified single clear bands in the species; from $29 \%$ to $75 \%$ of which were polymorphic to wheat. The frequency of amplification corresponded with their phylogenetic distance from barley. Many DNA markers (78-859) showing polymorphism between each species and wheat were obtained. These markers are expected to be valuable in identifying the alien chromosomes added to a wheat genetic background. The usefulness of the markers in the basic and the applied studies of the wild species is discussed.

Key Words: Hordeum vulgare, Triticum aestivum, Triticeae, chromosome addition lines, Expressed Sequence Tag, molecular marker, homoeologous group.

\section{Introduction}

The taxonomical tribe Triticeae, to which some important crops such as wheat and barley belong, is widely distributed in the world and possesses a great genetic variation. This variation could be utilized in breeding programs, as it is virtually possible to produce hybrids among various species in this tribe through embryo rescue. In polyploid wheat, for example, many lines with alien chromosomes or chromosome segments have been produced and some of them have been used in practical breeding programs (Friebe et al. 1996). In the process of developing these lines, a precise technique for detecting the alien chromosomes or chromosome segment(s) is essential. Availability of PCR-based molecular markers capable of detecting specific alien chromosomes or chromosomal fragments would enhance the use of the alien genetic variations in wheat breeding. However, the current limited knowledge on the genomes of wild Triticeae species does not provide the necessary DNA information for developing specific molecular markers for these species. This would be overcome by using the genome information of barley and wheat. Hernández et al. (2002) examined the transferability of wheat and barley simple sequence repeat

Communicated by K. Takeda

Received February 7, 2005. Accepted May 11, 2005.

*Corresponding author (e-mail: tsujim@muses.tottori-u.ac.jp) markers (SSRs) to the chromosomes of Hordeum chilense and demonstrate that these markers were a valuable resource for analyzing $H$. chilense chromosomes introduced to the genetic background of wheat.

Here we chose expressed sequence tag (EST) PCR primers of barley for the analysis of the alien species in Triticeae. EST resources provide sequences for developing a subcategory of sequence tagged site (STS) markers, which are known as expressed sequence tag-polymerase chain reaction (EST-PCR) markers. There are a number of advantages of using EST-PCR markers for genetic studies over randomprimed PCR markers. First, they originate from expressed sequences, and most of them can identify unique regions submerged in a large number of highly repetitive sequences, which are characteristic of the genomes of higher plants. Second, they are more likely to be conserved across species than non-coding regions, and thus to be useful for comparative mapping. Third, EST-PCR markers have the potential for being co-dominantly inherited, which allow the identification of two different alleles at heterozygous loci in diploid organisms (Schubert et al. 2001). However, considerable investment is required to develop these markers, and thus its production has been limited to economically important crops. If EST-PCR primers designed for crops could be used for other species, they would be of great value, especially for related alien species. The ability to transfer mapped STS markers within the Poaceae family has been demonstrated 
both between cereal species (Erpelding et al. 1996) and among cereal and forage grass species (Taylor et al. 2001).

Recently, a total of ca. 120,000 ESTs of barley strains 'Haruna Nijo', 'Akashinriki' and 'H602' (Hordeum vulgare ssp. spontaneum) were generated (Sato et al. 2003) in Okayama University, Japan. More than 60,000 cDNA sequences originating from the $3^{\prime}$ end were quality controlled and 10,336 primer sets were developed. Part of the information is available at http://www.shigen.nig.ac.jp/barley/. Using these markers for 93 doubled haploid lines between a barley cultivar 'Haruna Nijo' and the wild barley, H. spontaneum, Sato et al. (unpublished data) mapped about 2,000 fragments on the seven linkage groups.

The phylogenetic relationship in Triticeae has been resolved using morphological characters (Frederiksen and Seberg 1992), cytogenetic evidence (Wang 1992) and molecular data (Hsiao et al. 1995, Peterson and Seberg 1997, 2002 and Petersen et al. 2004). Morphological phylogenies exhibit high levels of homoplasy and the relationships of many taxa are unsolved. However, Hsiao et al. (1995) concluded that molecular data, morphological characters, and biogeographical information are complementary and are all essential for making meaningful phylogenetic inferences. Phylogeny using the internal transcribed spacer sequence clearly suggested that Thinopyrum is closely related to Triticum and Secale (Hsiao et al. 1995). Hordeum species are closely related each other specially the two species $H$. vulgare and H. bulbosum. Therefore, here we regarded Thinopyrum and $S$. cereale as wheat-related species. The selection criteria of species used in this study was firstly based on their contribution as chromosome donors to the addition lines in wheat and secondly to cover different genomic constitution, levels of ploidy, growth habitats, geographical distribution, and sexual reproduction systems.

In the present study, the applicability of 1,165 ESTbased barley PCR primer sets for assessment of polymorphisms between wheat and ten alien Triticeae species was examined. In addition, the usefulness of these markers in introducing the alien chromosomes in the genetic background of wheat and their application in breeding programs are discussed.

\section{Materials and Methods}

\section{Plant materials}

A total of 12 species of the tribe Triticeae were used (Table 1). The common wheat (Triticum aestivum) cv. 'Chinese Spring' as the recipient cultivar of most chromosome addition lines and barley (Hordeum vulgare) cv. 'Betzes' as the chromosome donor of the addition lines were used. These plant materials are being maintained in Tottori Alien Chromosome Bank of Wheat (TACBOW), managed by the National Bioresources Project-Wheat, Japan.

\section{DNA isolation and PCR}

Total genomic DNA was extracted from young plant leaves using the CTAB method (Murray and Thompson 1980). PCR was performed on a 96-well plate, with $10 \mu \mathrm{l}$ reaction volume containing $10 \mathrm{ng}$ template DNA, $5 \mathrm{pmol}$ of forward and reverse EST primers, $1 \mu \mathrm{l} 10 \mathrm{X}$ Ex-Taq buffer $\left(20 \mathrm{mM} \mathrm{Mg}^{2+}\right), 0.8 \mu \mathrm{l} \mathrm{dNTP}$ mixture $(2.5 \mathrm{mM}$ each) and 0.25 U Ex-Taq DNA polymerase (TaKaRa, Japan). After the initial denaturation step at $94^{\circ} \mathrm{C}$ for $2 \mathrm{~min}$, reactions were subjected to a touch-down amplification: 5 cycles at $94^{\circ} \mathrm{C}$ for 30 sec, $65^{\circ} \mathrm{C}$ for $30 \mathrm{sec}$ (decreasing by $1^{\circ} \mathrm{C} /$ cycle), $72^{\circ} \mathrm{C}$ for 2 min and subsequent 35 cycles at $94^{\circ} \mathrm{C}$ for $30 \mathrm{sec}, 60^{\circ} \mathrm{C}$ for 30 sec and $72^{\circ} \mathrm{C}$ for 2 min with a final extension step at $72^{\circ} \mathrm{C}$ for $7 \mathrm{~min}$. Electrophoresis of the PCR products was performed on $1.5 \%$ agarose gel. The presence of a clear band was scored as one unit and its absence or the presence of complicated types of amplifications scored as zero. The number of co-amplified marker units for each species was calculated pair wise. The following equation was used to calculate the difference between each pair:

$$
d=1-[a /\{(b+c)-a\}]
$$

where $d$ refers to the distance between the two species, $a$ refers to the number of co-amplified marker units, and $b$ and $c$ refer to the number of amplified marker units for the

Table 1. Genetic and ecological traits of the plant species used in the present study and availability of their addition lines in wheat

\begin{tabular}{|c|c|c|c|c|c|c|}
\hline Species & Accession No. & $\begin{array}{c}\text { Genome } \\
\text { constitution }\end{array}$ & $\begin{array}{l}\text { Chromosome } \\
\text { number }(2 n)\end{array}$ & $\begin{array}{l}\text { Growth } \\
\text { habit }\end{array}$ & $\begin{array}{l}\text { Availability of } \\
\text { addition lines }\end{array}$ & Reference \\
\hline Hordeum vulgare cv. 'Betzes' & TACBOW0116 & $\mathrm{HH}^{1)}$ & 14 & Annual & $1 \mathrm{H}-7 \mathrm{H}$ & Islam et al. (1981) \\
\hline H. bulbosum & TACBOW0117 & $\mathrm{HH}^{1)}$ & 14 & Perennial & Not available & - \\
\hline H. chilense & TACBOW0115 & $\mathrm{HH}$ & 14 & Perennial & $1-2 \mathrm{H}^{\mathrm{ch}}, 4-7 \mathrm{H}^{\mathrm{ch}}$ & Miller et al. (1982) \\
\hline H. bogdanii & TACBOW0118 & $\mathrm{HH}$ & 14 & Perennial & Not available & - \\
\hline Dasypyrum villosum & TACBOW0119 & VV & 14 & Annual & $1 \mathrm{~V}-6 \mathrm{~V}$ & Sears (1953) \\
\hline Elymus ciliaris & TACBOW0122 & StStYY & 28 & Perennial & Partially produced & Sharma and Gill (1983) \\
\hline Psathyrostachys huashanica & TACBOW0121 & NsNs & 14 & Perennial & $1 \mathrm{Ns}-7 \mathrm{Ns}$ & Unpublished \\
\hline Leymus racemosus & TACBOW0111 & NsNsXmXm & 28 & Perennial & 10 lines & Kishii et al. (2004) \\
\hline L. mollis & TACBOW0113 & NsNsXmXm & 28 & Perennial & 10 lines & Unpublished \\
\hline Secale cereale strain 'IR90' & HT23S33 & $\mathrm{RR}$ & 14 & Annual & $1 \mathrm{R}-7 \mathrm{R}$ & Unpublished \\
\hline Thinopyrum elongatum & TACBOW0120 & EE & 14 & Perennial & $1 \mathrm{E}-7 \mathrm{E}$ & Dvořák and Knott (1974) \\
\hline Triticum aestivum cv. 'Chinese Spring, & HT23S1 & AABBDD & 42 & Annual & - & - \\
\hline
\end{tabular}

1) Following recommendations for the designation of the barley chromosomes and their arms at the business meeting of the 7 th International Barley Genetics Symposium held at the University of Saskatchewan, Saskatoon, Saskatchewan, Canada, on August 5, 1996. 
first and second species, respectively. Unweighted pair-group method arithmetic average (UPGMA) clustering, NTSYSpcversion 2.1 (Rohlf 2000) was used to construct a tree dendrogram.

\section{EST primers}

Four series of barley EST primer sets were examined. Series 1 consisted of 384 primer sets that were chosen randomly from the pool of the barley EST primer sets. Series 2, 3 and 4 were pre-screened in the previous study (Sato et al. 2003). Series 2 consisted of 651 primer sets that showed a clear single band in barley but not in wheat. Series 3 consisted of 42 primer sets that showed a clear single band with a different size in both barley and wheat whereas series 4 consisted of 88 primers that showed a clear single band of the same size. The four series originated from 10,336 ESTs markers which were produced on the bases of the ESTs information of barley cultivars 'Haruna Nijo', 'Akashinriki' and wild barley, $H$. vulgare ssp. spontaneum strain 'H602'. More information is available at http://www.shigen.nig.ac.jp/ barley/.

\section{Results}

\section{Applicability of barley EST primers to the other Triticeae species}

The number of amplified fragments differed with the species and the primer series. There were two types of presence/absence polymorphism between wheat and the alien species. In this study we considered only the presence of the

Table 2. Number and frequency of amplified markers by four barley EST primer series (1,165 primers in total) in the 12 studied Triticeae species

\begin{tabular}{|c|c|c|c|c|c|c|c|c|c|c|}
\hline \multirow{3}{*}{$\begin{array}{l}\text { Species } \\
\text { H. vulgare }\end{array}$} & \multicolumn{8}{|c|}{ No. of amplified markers (\%) } & \multirow{2}{*}{\multicolumn{2}{|c|}{$\begin{array}{c}\text { Total No. of amplified } \\
\text { markers }(\%)\end{array}$}} \\
\hline & \multicolumn{2}{|c|}{ Series $1^{1)}$} & \multicolumn{2}{|c|}{ Series $2^{2)}$} & \multicolumn{2}{|c|}{ Series $3^{3)}$} & \multicolumn{2}{|c|}{ Series $4^{4)}$} & & \\
\hline & 364 & $(94.8)^{5)}$ & 651 & $(100)$ & 42 & $(100)$ & 88 & $(100)$ & 1145 & (98.2) \\
\hline H. bulbosum & 293 & $(76.3)$ & 414 & $(63.6)$ & 39 & $(92.9)$ & 74 & $(84.1)$ & 820 & $(70.4)$ \\
\hline H. chilense & 216 & $(56.3)$ & 198 & $(30.4)$ & 32 & $(76.2)$ & 64 & $(72.7)$ & 510 & $(43.8)$ \\
\hline H. bogdanii & 165 & $(43.0)$ & 150 & $(23.0)$ & 17 & $(40.5)$ & 58 & $(65.9)$ & 390 & $(33.5)$ \\
\hline D. villosum & 152 & $(39.6)$ & 91 & $(14.0)$ & 11 & $(26.2)$ & 63 & (71.6) & 317 & $(27.2)$ \\
\hline E. ciliaris & 193 & $(50.3)$ & 123 & (18.9) & 14 & (33.3) & 63 & (71.6) & 393 & $(33.7)$ \\
\hline L. mollis & 209 & $(54.4)$ & 147 & $(22.6)$ & 27 & $(64.3)$ & 61 & $(69.3)$ & 444 & $(38.1)$ \\
\hline L. racemosus & 196 & $(51.0)$ & 122 & $(18.7)$ & 14 & (33.3) & 57 & $(64.8)$ & 389 & (33.4) \\
\hline P. huashanica & 153 & $(39.8)$ & 96 & $(14.7)$ & 17 & $(40.5)$ & 42 & $(47.7)$ & 308 & (26.4) \\
\hline S. cereale & 152 & $(39.6)$ & 47 & (7.2) & 9 & $(21.4)$ & 50 & $(56.8)$ & 258 & $(22.1)$ \\
\hline Th. elongatum & 172 & $(44.8)$ & 39 & $(6.0)$ & 10 & $(23.8)$ & 50 & $(56.8)$ & 271 & $(23.3)$ \\
\hline T. aestivum & 200 & $(52.1)$ & & - & 42 & $(100)$ & 88 & $(100)$ & 330 & $(64.2)$ \\
\hline Average & 205.4 & $(53.5)$ & 188.9 & $(29.0)$ & 22.8 & $(54.4)$ & 63.2 & $(71.8)$ & 464.6 & $(39.9)$ \\
\hline
\end{tabular}

1) Primer series 1 consists of 384 barley primer sets that were chosen randomly.

2) Primer series 2 consists of 651 barley primers that showed a single band in barley but not in wheat in a previous study.

3) Primer series 3 consists of 42 barley primers that showed single band with different size in barley and wheat in a previous study.

4) Primer series 4 consists of 88 barley primers that showed a single band with the same size in barley and wheat in a previous study.

5) Number in parentheses is percentage of number of the amplified fragments for the species to total number of tested markers in each series.

Table 3. Number and frequency of polymorphic EST markers to that in wheat

\begin{tabular}{|c|c|c|c|c|c|c|}
\hline \multirow{3}{*}{$\begin{array}{l}\text { Species } \\
\text { H. vulgare }\end{array}$} & \multicolumn{2}{|c|}{$\begin{array}{l}\text { No. of markers amplified in alien } \\
\text { species but not in wheat }(\%)\end{array}$} & \multicolumn{2}{|c|}{$\begin{array}{c}\text { No. of markers of polymorphism } \\
\text { to wheat }(\%)\end{array}$} & \multirow{2}{*}{\multicolumn{2}{|c|}{$\begin{array}{c}\text { Total No. of markers of } \\
\text { polymorphism to wheat } \\
(\%)\end{array}$}} \\
\hline & Series 1 & Series 2 & Series 3 & Series 4 & & \\
\hline & $166(45.6)^{1)}$ & $651 \quad(100)$ & $42 \quad(100)$ & - & 859 & $(75.0)$ \\
\hline H. bulbosum & 119 (40.6) & $414 \quad(100)$ & $31 \quad(79.5)$ & $8 \quad(10.8)$ & 572 & (69.8) \\
\hline H. chilense & $62 \quad(28.7)$ & $198 \quad(100)$ & $22 \quad(68.8)$ & $10 \quad(15.6)$ & 292 & (57.3) \\
\hline H. bogdanii & $38 \quad(23.0)$ & $150 \quad(100)$ & 11 (64.7) & $6 \quad(10.3)$ & 205 & $(52.6)$ \\
\hline D. villosum & $30 \quad(19.7)$ & $91 \quad(100)$ & $9 \quad(81.8)$ & $(3.2)$ & 132 & $(41.6)$ \\
\hline E. ciliaris & $42 \quad(21.8)$ & $123 \quad(100)$ & $10 \quad(71.4)$ & $(6.3)$ & 179 & $(45.5)$ \\
\hline L. mollis & $50 \quad(23.9)$ & $147 \quad(100)$ & $14 \quad(51.9)$ & $13 \quad(21.3)$ & 224 & $(50.5)$ \\
\hline L. racemosus & $46 \quad(23.5)$ & $122(100)$ & $10 \quad(71.4)$ & $(7.0)$ & 182 & $(46.8)$ \\
\hline P. huashanica & $36 \quad(23.5)$ & $96 \quad(100)$ & $10 \quad(58.8)$ & $7 \quad(16.7)$ & 149 & $(48.4)$ \\
\hline S. cereale & $26 \quad(17.1)$ & $47 \quad(100)$ & $6 \quad(66.7)$ & $(6.0)$ & 82 & (31.8) \\
\hline Th. elongatum & $29 \quad(16.9)$ & $39 \quad(100)$ & $4 \quad(40.0)$ & $6 \quad(12.0)$ & 78 & $(28.8)$ \\
\hline Average & $58.5 \quad(28.5)$ & $188.9 \quad(100)$ & $15.4 \quad(67.3)$ & $6.3 \quad(10.0)$ & 268.5 & $(56.3)$ \\
\hline
\end{tabular}

1) Number in parentheses is percentage of number of the polymorphic markers (to wheat) to number of amplified markers for each species as in Table 2. 
marker in alien species and its absence in wheat. Amplification of markers in wheat but not in alien species is not of interest, as we want to use these markers to detect alien DNA in the wheat genetic background. Percentages of primer sets that produced a single clear band in the studied species ranged from $22.5 \%$ to $100 \%$ (Table 2). Moreover, 28.8$75.0 \%$ of the amplified fragments showed either presence/ absence or size polymorphisms between the species and wheat (Table 3 ).

A considerable number of single clear bands were produced in all the species using the primer sets of series 1 (Fig. 1 and Table 2). In addition to these single bands, faint multiple bands often appeared. These faint multiple bands may be attributed to non-homologous amplification due to the lack of homologous sequences in the genomes. Of the 384 primer sets in series 1, 11 primer sets failed to amplify bands with any of the studied species even with barley cultivar 'Betzes' (Fig. 2). Interestingly, 9 primers sets produced the amplified fragment in some species but not barley. This may be due to difference in PCR condition and/or difference in barley cultivar from those used to develop the primer sets. The present barley cultivar 'Betzes' showed the highest number (364) of bands. Barley-related species (H. bulbosum and $H$. chilense) and polyploid species (L. mollis, T. aestivum, L. racemosus, and E. ciliaris) showed more bands than the other species i.e., 293, 216, 209, 200, 196 and 193 bands, respectively. An exception was $H$. bogdanii, which belongs to the same genus of barley, but the number of amplified bands was considerably low (165 bands).

Markers showing polymorphism between wheat and the alien species were needed to detect alien chromosome(s) in the wheat genetic background. Thus, in the second experiment we used primer series that had already been prescreened by the PCR with positive amplification in barley and negative in wheat (Sato et al. 2003). Most of the 651 primer sets in series 2 that showed a clear single band in barley but not in wheat produced a single clear band in certain species while multiple faint bands in the others. These faint bands have not been counted as positive amplification units. Some primer sets could not amplify any PCR products in some species. From 39 to 651 markers for each species were obtained (Table 2). More markers were obtained in the Hordeum species than in any of the other species.

To detect the size polymorphic markers between wheat and the alien species we used primer series 3 that produced a single band with a different sizes in both barley and wheat and primer series 4 that produced a single band with the same size in the pre-screening. Applying primers in series 3, we obtained from 9 to 42 markers. Primers in series 4 produced from 42 to 88 markers in the studied species (Table 2).

In general, the average percentage of the fragments showing polymorphism between wheat and each species (Table 3) were higher in primers series $2(100 \%)$ and 3 $(67.3 \%)$ than that in series $1(28.5)$ and $4(10.0 \%)$. We obtained a total of 2,954 markers showing polymorphism between wheat and the other species. The total number of poly-
Primer

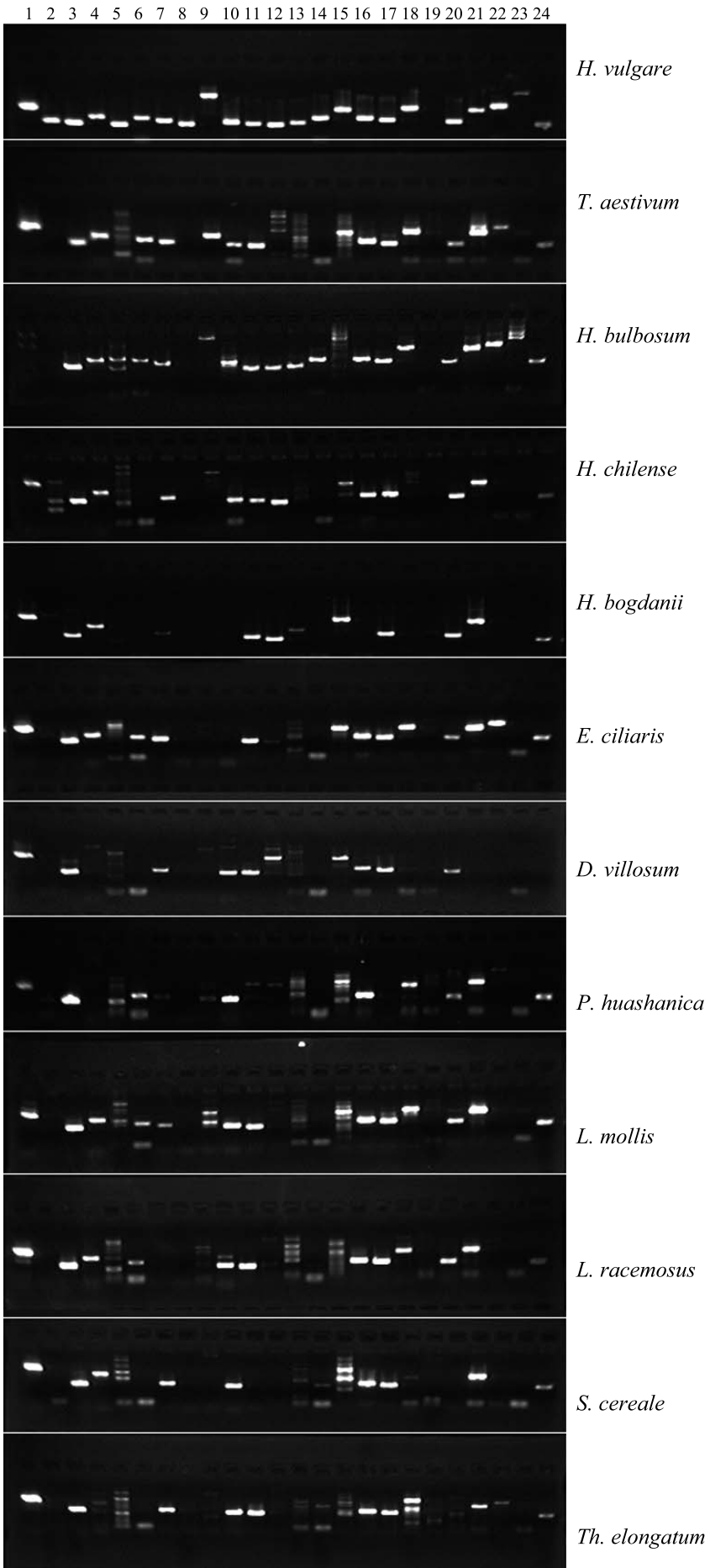

Fig. 1. Representative images of PCR amplification patterns of the 12 Triticeae species using 24 barley EST primer sets. Electrophoresis of the PCR products was performed on $1.5 \%$ agarose gel.

morphic markers showed a great variation among the species. $H$. vulgare, $H$. bulbosum and $H$. chilense had the highest number of markers showing polymorphism with wheat and ranged from 859 to 292 markers. L. mollis, 


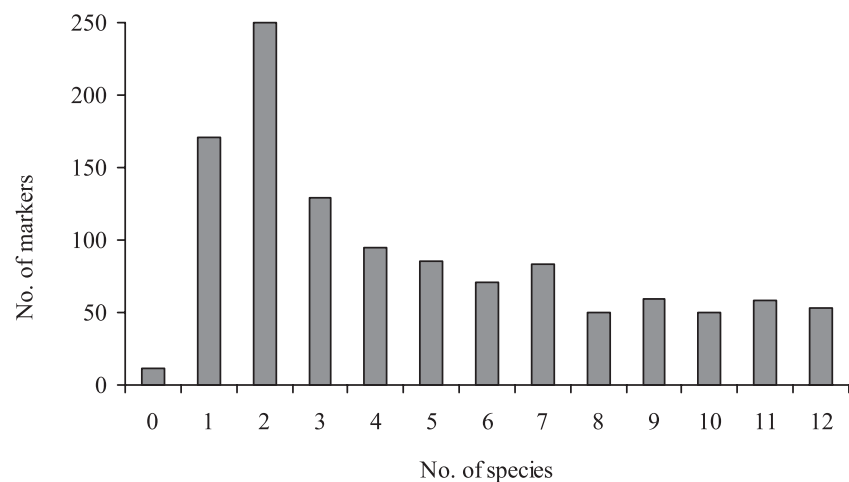

Fig. 2. Frequency of co-amplified fragments among 12 Triticeae species using 1,165 barley EST primer sets.

L. racemosus, E. ciliaris, P. huashanica, and D. villosum showed 224, 182, 179, 149 and 132 markers, respectively. Meanwhile, S. cereale and Th. elongatum had the lowest number of markers (82 and 78 markers, respectively).

\section{Co-amplification of barley EST markers among species in Triticeae}

Most primer sets produced the amplified fragment in barley as well as in the other species. A total of 1,165 barley EST primer sets were used to calculate the degree of coamplification between each two species (Table 4). The frequency of co-amplification of barley EST markers in the present species is shown in Fig. 2. There were 171 markers amplified only in barley. In barley and one of the alien species, mainly $H$. bulbosum, 250 markers were co-amplified; 53 markers were co-amplified in all 12 species (Fig. 2). Of the primer sets that amplified a clear band in barley $(1,145$ primers in total), $71.6 \%$ produced the amplified fragment in H. bulbosum, whereas, $22.5 \%$ and $23.7 \%$ produced the amplified fragment in $S$. cereale and Th. elongatum, respectively. This frequency seemed to correlate well with the phylogenetic relationship of the species with barley. This was evident from the dendrogram drawn based on the coamplification frequency (Fig. 3).

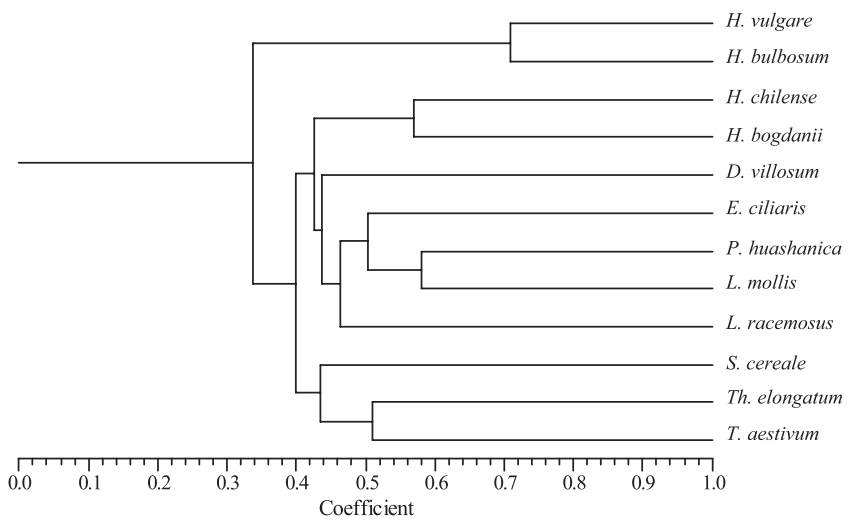

Fig. 3. Dendrogram generating from results of PCR amplifications of 12 Triticeae using 1,165 barley EST primer sets. The unweighted pair group method with arithmetic mean was used as clustering method.

\section{Discussion}

The aim of this study is to obtain markers showing polymorphism between the alien species and wheat. Therefore, barley EST information was used, instead of wheat ESTs, to produce markers for identifying alien chromosomes in the wheat genetic background. Sato et al. (2003) reported that 1,694 out of 5,133 markers $(33.0 \%)$ showed presence/absence type of polymorphism between barley and wheat. We tested four series of barley EST primers in this study; the first series consisted of randomly chosen EST primers, while the remaining three series consisted of prescreened primers showing presence/absence type of polymorphism and co-amplified fragments with the same or different size between wheat and barley. For the four series, the frequency of markers showing polymorphism between wheat and the alien species was found to be correlated with the phylogenetic relationship with barley.

In this study, a total of 1,165 primer sets with average size of 20 base pairs were examined. This implies that the homology of 46,600 base pairs $(1,165 \times 20 \times 2)$ was determined by PCR analysis. It would be difficult to directly discuss the phylogeny of the species based on the data since several factors are involved in the annealing between the

Table 4. Number of the markers co-amplified among 12 Triticeae species using 1,165 barley EST primer sets

\begin{tabular}{|c|c|c|c|c|c|c|c|c|c|c|c|}
\hline & H.vul. & H.bul. & H.chi. & H.bog. & D.vil. & E.cil. & L.mol. & L.rac. & P.hua. & S.cer. & Th.elo. \\
\hline H. bulbosum & 815 & & & & & & & & & & \\
\hline H. chilense & 505 & 441 & & & & & & & & & \\
\hline H. bogdanii & 387 & 350 & 327 & & & & & & & & \\
\hline D. villosum & 313 & 277 & 243 & 208 & & & & & & & \\
\hline E. ciliaris & 389 & 340 & 278 & 235 & 237 & & & & & & \\
\hline L. mollis & 441 & 384 & 324 & 251 & 224 & 283 & & & & & \\
\hline L. racemosus & 385 & 340 & 291 & 228 & 220 & 259 & 306 & & & & \\
\hline P. huashanica & 308 & 265 & 222 & 182 & 171 & 208 & 246 & 226 & & & \\
\hline S. cereale & 256 & 222 & 203 & 164 & 173 & 191 & 199 & 186 & 154 & & \\
\hline Th. elongatum & 270 & 235 & 213 & 177 & 164 & 198 & 201 & 189 & 146 & 154 & \\
\hline T. aestivum & 330 & 287 & 250 & 202 & 196 & 228 & 247 & 221 & 176 & 185 & 203 \\
\hline
\end{tabular}


primers and the genomic DNA. However, the dendrogram drawn from the present data did not deviate largely from the real feature of their phylogeny (Fig. 3) (Hsiao et al. 1995, Peterson and Seberg 1997, 2002 and Petersen et al. 2004).

The huge variation in Triticeae is a potential genetic resource for breeding of cultivated species within the same tribe, especially common wheat. However, the removal of deleterious genes located on the same alien chromosome is necessary before introducing the lines to practical breeding. Only small chromosome segments with useful gene(s) must be transferred to the wheat chromosome by homoeologous chromosome recombination using the phl gene or its suppressor $P h^{I}$ (Sears 1977, Chen et al. 1994, AghaeeSarnarzeh et al. 2000) or by translocation using irradiation (Sears 1967, 1993, Tsujimoto et al. 2001). PCR markers located on the targeted segment would be helpful for following the desired chromosome segment with useful trait. This study showed that barley EST markers could be used to identify alien chromosomes of Triticeae. A considerable number of markers (78-859) were obtained for each species (Table 3). Because the genomes of the Triticeae consist of seven chromosomes, this number implies the presence of 11-122 markers on average for each chromosome. Using the sets of markers for the same alien chromosomes, the processes of homoeologous recombination and translocation could be followed.

Most barley ESTs have already been localized on the rice linkage map by sequence homology comparisons between their original barley ESTs and the public rice genome information (http://earth.lab.nig.ac.jp/ dclust/cgi-bin/barley_ map_pub/index.html). Via barley ESTs the genome information of Triticeae species can be linked to rice, a model plant, in which the genome sequence has been completed. The very limited information for genome research in the minor Triticeae species can be partly overcome by using the barley EST markers. Many of these markers have already been marked on the fine linkage map of barley. This allows us to identify the part(s) of alien chromosomes homoeologous to the barley chromosome, and to trace back the chromosome evolution to allocate the markers on the alien chromosomes. The concept of using barley EST primers in the development of markers proposed here is expected to promote the use of variations in alien species of Triticeae for practical breeding programs in wheat. Further studies are in progress to allocate these barley EST markers on each alien chromosome added in wheat and to isolate broken alien chromosome(s) by the Gc gene-system (Endo and Gill 1996) and heavy ion irradiation (Tsujimoto et al. 2001). These chromosomes will reveal more information on the homoeologous relationship in the genomes of Triticeae.

\section{Acknowledgment}

The primers used for this study were provided by the Core Research for Evolutional Science and Technology, Japan Science and Technology Corporation.

\section{Literature Cited}

Aghaee-Sarnarzeh,M., Harjit-Singh and H.S.Dhaliwal (2000) $P h^{I}$ gene derived from Aegilops speltoides induce homoeologous chromosome pairing in wide crosses of Triticum aestivum. J. Hered. 91: 417-421.

Chen,P.D., H.Tsujimoto and B.S.Gill (1994) Transfer of $P h^{I}$ genes promoting homoeologous pairing from Triticum speltoides to common wheat. Theor. Appl. Genet. 88: 97-101.

Dvořák, J. and D.R.Knott (1974) Disomic and ditelosomic addition lines of diploid Agropyron elongatum chromosomes to Triticum aestivum. Can. J. of Genet. Cytol. 16: 399-417.

Endo,T.R. and B.S.Gill (1996) The deletion stocks of common wheat. J. Hered. 87: 295-307.

Erpelding,J.E., N.K.Blake, T.K.Blake and L.E.Talbert (1996) Transfer of sequence tagged site PCR markers between wheat and barley. Genome 39: 802-812.

Frederiksen,S. and O.Seberg (1992) Phylogenetic analysis of Triticeae (Poaseae). Hereditas 116: 15-19.

Friebe, B., J.Jiang, W.J.Raupp, R.A.McIntosh and B.S.Gill (1996) Characterization of wheat-alien translocations conferring resistance to diseases and pests: current status. Euphytica 91: 59-87.

Hernández,P., D.A.Laurie, A.Martín and J.W.Snape (2002) Utility of barley and wheat simple sequence repeat (SSR) markers for genetic analysis of Hordeum chilense and tritordeum. Theor. Appl. Genet. 104: 735-739.

Hsiao, C., N.J.Chatterton, K.H.Asay and K.B.Jensen (1995) Phylogenetic relationships of the monogenomic species of the wheat tribe, Triticeae (Poaceae), inferred from nuclear rDNA (internal transcribed spacer) sequences. Genome 38: 211-223.

Islam,A.K.M.R., E.W.Shepherd and D.H.B.Sparrow (1981) Isolation and characterization of euplasmic wheat-barley chromosome addition lines. Heredity 46: 161-174.

Kishii,M., T.Yamada, T.Sasakuma and H.Tsujimoto (2004) Production of wheat-Leymus racemosus chromosome addition lines. Theor. Appl. Genet. 109: 255-260.

Miller,T.E., S.M.Reader and V.Chapman (1982) The addition of Hordeum chilense chromosomes to wheat. In "Induced Variability in Plant Breeding: Proceedings of an International Eucarpia Symposium” Broertjes, C. (ed.), Pudoc, Wageningen, The Netherlands, p. 79-81.

Murray,H.G. and W.F.Thompson (1980) Rapid isolation of high molecular weight plant DNA. Nuc. Acid Res. 8: 4321-4325.

Peterson, G. and O.Seberg (1997) Phylogenetic analysis of the Triticeae (Poaceae) based on rpoA sequence data. Mol. Phylogenet. Evol. 7: $217-230$.

Peterson, G. and O. Seberg (2002) Molecular evolution and phylogenetic application of DMC1. Mol. Phylogenet. Evol. 22: 43-50.

Petersen, G., O.Seberg, L.Aagesen and S.Frederiksen (2004) An empirical test of the treatment of indels during optimization alignment based on the phylogeny of the genus Secale (Poaceae). Mol. Phylogenet. Evol. 30: 733-742.

Rohlf,F.J. (2000) "NTSYS-pc numerical taxonomy and multivariate analysis system, version 2.1" Exeter Publication, New York.

Sato, K., N.Nankaku, K. Yano and K. Takeda (2003) Large scale development of barley EST markers. Ikushugaku Kenkyu, 5 (suppl. 1): 93.

Schubert,R., G.Mueller-Starck and R.Riegel (2001) Development of EST-PCR markers and monitoring their intrapopulational genetic variation in Picea abies (L.) Karst. Theo. Appl. Genet. 103: $1223-1231$. 
Sears,E.R. (1953) Addition of the genome of Haynaldia villosa to Triticum aestivum. Am. J. Bot. 40: 168-174.

Sears,E.R. (1967) Induced transfer of hairy neck from rye to wheat. Zeitschrift für Pflanzenzuchtung 57: 4-25.

Sears,E.R. (1977) An induced mutant with homoeologous pairing in common wheat. Can. J. Genet. Cytol. 19: 585-593.

Sears,E.R. (1993) Use of radiation to transfer alien chromosome segments to wheat. Crop Sci. 33: 897-901.

Sharma,H.C. and B.S.Gill (1983) New hybrid between Agropyron and wheat. III. Backcross derivatives, effect of Agropyron cytoplasm, and production of addition lines. In "Proc. 6th Int.
Wheat Genet. Symp.” Sakamoto, S. (ed.). Plant Germ-plasm Institute, Kyoto University, Japan, p. 213-221.

Taylor,C., K.Madsen, S.Borg, M.G.Moller, B.Boelt and P.B.Holm (2001) The development of sequence-tagged sites (STSs) in Lolium perenne L.: The application of primer sets derived from other genera. Theor. Appl. Genet. 103: 648-658.

Tsujimoto,H, T.Yamada and T.Abe (2001) Chromosome breakage in wheat induced by heavy ion-beam irradiation. Riken Report 34: 172 .

Wang, R.R.-C. (1992) Genomic relationships in perennial Triticeae based on diploid hybrids and beyond. Hereditas 116: 133-136. 